> La montée en puissance du marché biopharmaceutique génère des besoins énormes en production de molécules thérapeutiques. Les capacités mondiales de production, principalement de protéines thérapeutiques, impossible à obtenir par synthèse chimique, sont actuellement insuffisantes. Outre les aspects économiques en jeu, la question des technologies utilisées pour produire ces molécules est importante: les technologies éprouvées (bactéries, levures, cellules de mammifères), utilisées depuis longtemps assez largement, restent coûteuses ou voient leur champ d'application limité ; les technologies innovantes (cellules aviaires, animaux et végétaux transgéniques, entre autres), quant à elles, doivent encore faire leurs preuves. <

\section{Bioproduction de protéines thérapeutiques}

\section{Revue et perspectives}

Joëlle Dumas, Bénédicte Robert

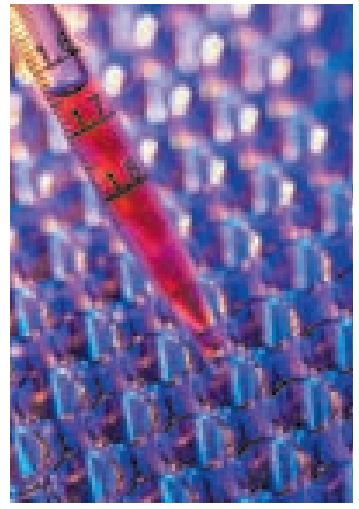

Genopole Campus 1, 5 , rue Henri Desbruères, 91030 Évry Cedex, France. benedicte.robert@ genopole.fr joelle.dumas@genopole.fr

et 126 en phase III. Cette étude dresse également un état des lieux des implantations d'unités de bioproduction en Europe et dans le reste du monde, et hiérarchise les critères de décision d'installation de ces unités. "Les facteurs clés d'implantation majeurs identifiés par l'étude sont les politiques fiscale et sociale du pays d'accueil, la formation et l'expérience professionnelle, ainsi que l'environnement technique et scientifique», précise Pierre Tambourin, directeur général de Genopole ${ }^{\circledR}$.

«Un pays développé doit pouvoir faire bénéficier sa population de ces nouvelles voies de traitement. Il en va de son indépendance sanitaire, de sa capacité à anticiper les évolutions épidémiologiques nationales et à figurer en bonne place dans le «club» très envié des grands pays leaders en matière de santé», ajoute Christian Lajoux, président du LE\&M.

\section{La production de protéines thérapeutiques}

À l'origine, les protéines d'intérêt thérapeutique étaient extraites de sources naturelles telles que le sang, le placenta, ou autres extraits de tissus humains ou animaux. Toutefois, les quantités restaient limitées, et les produits potentiellement dangereux pour les patients, du fait de risques de contamination par des virus - Sida, hépatite $B$ notamment, comme ce fut le cas dans l'affaire du sang contaminé - ou des prions, qui furent notamment en cause dans le déve- 
loppement de la maladie de Creutzfeldt-Jakob chez des patients traités à l'hormone de croissance d'origine humaine.

L'essor de la génomique et des technologies de I'ADN recombinant dans les années 1970 a permis le développement de nouveaux procédés de production de protéines, mettant en œuvre divers systèmes d'expression. L'identification, puis le clonage des gènes gouvernant la synthèse des principales protéines humaines d'intérêt thérapeutique ont rendu possible la production de celles-ci par des organismes hétérologues, et ces protéines, dites recombinantes, ont constitué une alternative aux protéines d'extraction. Aujourd'hui, la quasi-totalité des protéines thérapeutiques produites sont des protéines recombinantes.

En moins de 30 ans, les technologies qui se sont développées depuis le début des années 1980 ont atteint une maturité industrielle et commerciale qui n'est aujourd'hui plus contestée. Toutes les grandes entreprises pharmaceutiques ont investi massivement dans ces technologies, qui ont prouvé leur capacité à produire à grande échelle des protéines thérapeutiques efficaces et sécuritaires pour les patients. Ces technologies de production sont donc promises à un bel avenir porté par un marché des protéines thérapeutiques en forte croissance. En revanche, les systèmes biologiques étant par nature beaucoup plus difficiles à contrôler que les procédés de production chimiques, les rendements variables, ainsi que la pureté et la sécurité, resteront des défis importants associés à ces technologies. Par ailleurs, celles-ci nécessitent des investissements considérables en capital et génèrent des coûts d'opérations élevés. Ceci augmente de façon significative les coûts par dose comparés aux médicaments chimiques. Avec une pression grandissante sur les coûts de santé, c'est probablement le plus grand défi auquel ces technologies devront faire face dans l'avenir.

Avec une gamme de fonctions biologiques très large, les protéines sont communément utilisées pour de multiples applications médicales, résumées dans le Tableaul.

La première classe de protéines thérapeutiques, les anticorps monoclonaux, découverts il y a 30 ans, sont devenus les produits pharmaceutiques qui ont le plus fort taux de développement pour de nouvelles applications thérapeutiques, notamment pour le traitement du cancer. Leur production industrielle est en pleine croissance, mais demeure encore très coûteuse et aura bientôt du mal à répondre aux demandes

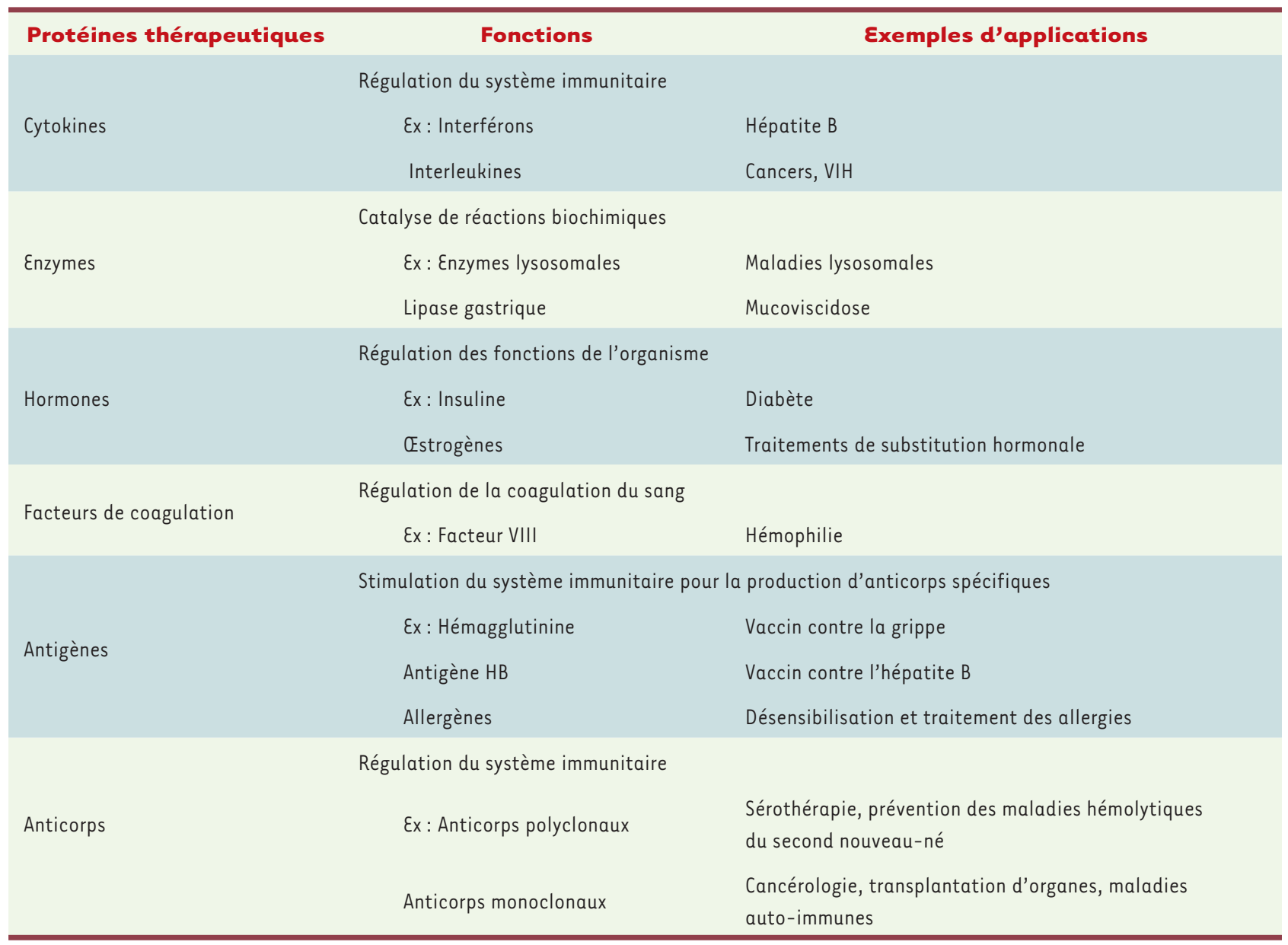

Tableau I. Applications médicales des protéines thérapeutiques. 
de grosses quantités pour les candidats médicaments en fin de phase III.

Les protéines recombinantes sont produites à partir d'un transgène introduit dans un organisme hétérologue par génie génétique. Ce procédé biotechnologique se déroule suivant cinq grandes étapes :

1. la construction d'un vecteur d'expression (en général, plasmide ou virus) jouant le rôle de transporteur du gène codant pour la protéine d'intérêt;

2. la modification d'une cellule hôte ou d'un organisme complexe par le vecteur d'expression;

3. la production de ce nouveau système d'expression, qui exécute les instructions fournies par le transgène, en l'occurrence synthétise la protéine d'intérêt;

4. la séparation de la protéine du milieu de culture, quand elle est secrétée, ou son extraction du milieu intracellulaire le cas échéant, puis sa purification;

5. la caractérisation de la molécule et la vérification de son degré de pureté.

\section{Des défis majeurs}

Les principaux défis des acteurs de la bioproduction pharmaceutique en vue de l'abaissement des coûts et des délais de production sont de trois ordres:

- loptimisation du système d'expression des molécules - amélioration de la productivité des clones et des techniques et rendements de production en masse, mise au point de milieux de culture exempts de sérum bovin (éliminant ainsi les risques de contamination, notamment par les prions), optimisation des profils de glycosylation;

- l'excellence opérationnelle, par l'identification et l'actionnement des différents bras de levier de l'amélioration des performances industrielles: amélioration des performances et rendements en downstream process (extraction/purification des molécules), développement des Process analytical technologies (PAT, techniques analytiques en ligne) pour limiter les coûts et délais des contrôles en cours de process, développement des technologies de production à usage unique pour limiter les coûts et délais inféodés aux procédés connexes à la production (nettoyage et stérilisation notamment) et aux actions d'assurance-qualité associées (qualification des équipements, validation des procédés connexes...), optimisation des flux et des cycles de production...;

- une meilleure compréhension des mécanismes d'action des biomolécules et de l'impact de leur structure tri-dimensionnelle sur leur activité, en vue d'orienter le choix du modèle d'expression en fonction de l'application thérapeutique ciblée.

\section{Des technologies éprouvées encore coûteuses...}

Une large gamme de systèmes d'expression est aujourd'hui disponible, chacun d'eux présentant des avantages et des inconvénients. Les protéines recombinantes actuellement commercialisées sont produites majoritairement par des bactéries, levures, cellules de mammifères et œufs embryonnés.

\section{Levures et bactéries}

- Depuis l'utilisation de «bactéries recombinantes» pour produire l'insuline et l'hormone de croissance au début des années 1980, cette technologie a été largement utilisée, mais a également montré ses limites. $\varepsilon n$ effet, les bactéries sont incapables de produire des protéines ayant une structure complexe comme les anticorps ou les facteurs de coagulation sanguine. Pour être stables et actives in vivo et donc efficaces chez l'homme, ces protéines doivent subir de multiples modifications post-traductionnelles propres aux mammifères : repliement, clivage, association de sousunités, $\gamma$-carboxylation et glycosylation, mécanismes métaboliques complexes que les procaryotes ne mettent pas en œuvre, ce qui limite leur utilisation à la production de biomolécules simples (petits polypeptides). Par ailleurs, il est souvent nécessaire de lyser la bactérie pour récupérer une molécule qui n'est généralement pas sécrétée, ce qui complexifie le downstream process (extraction/purification) et nuit aux rendements de purification.

- Les levures et champignons filamenteux offrent les mêmes facilités expérimentales et industrielles que les bactéries. Eucaryotes inférieurs, ils donnent accès à une plus large gamme de protéines recombinantes, dans la mesure où ils réalisent un certain nombre de modifications post-traductionnelles, notamment des glycosylations simples, la formation de ponts disulfures..., mais ils peuvent produire simultanément des sucres indésirables, conduisant à l'hypermannosylation de la protéine d'intérêt, la rendant immunogène. Ils se sont révélés peu adaptés pour la production des protéines les plus complexes, comme les anticorps monoclonaux. Citons cependant une application thérapeutique majeure, la production de vaccins commerciaux contre I'hépatite B (antigène HB) par Saccharomyces cerevisiae. Des levures génétiquement modifiées, exprimant un gène codant pour la glycosylation, se sont montrées capables de secréter des protéines recombinantes très semblables aux molécules humaines; ceci porte à croire que les levures peuvent devenir un système de production essentiel dans les années à venir.

Pour la production de molécules simples, les bactéries et les levures restent très compétitives, du fait de la parfaite maîtrise de leur production industrielle et de par leur riche historique dans l'industrie agroalimentaire.

\section{Cellules de mammifères}

Le système d'expression en cellules de mammifères reste actuellement le plus utilisé. Cinquante pour cent des anticorps monoclonaux produits aujourd'hui le sont 
par des cellules de mammifères, les cellules de hamster principalement ( $\mathrm{CHO}$ : Chinese hamster ovary; BHK: baby hamster kidney), et dans une moindre mesure les cellules murines (cellules NSO); les cellules humaines font leur apparition avec, entre autres, les cellules de rétine (cellules PER.C6), des cellules cancéreuses du col de l'utérus (HeLa), les lymphocytes T (Jurkat), qui présentent l'énorme avantage de la glycosylation humaine.

Pour la production de vaccins, l'éventail de modèles cellulaires est plus large, comptant notamment des lignées cellulaires de rein canin (Madin-Darby canine kidney, MDCK), de rein de singe (cellules Vero de singe vert africain) ou de rétine humaine (cellules PER.C6).
Le principal avantage de ce système est que les protéines, sécrétées dans le milieu de culture, ont subi les modifications post-traductionnelles propres aux mammifères. Les inconvénients majeurs restent la faible capacité de production, de l'ordre de quelques centaines de milliers de litres de bioréacteurs à l'échelle de la planète, des investissements industriels et des coûts de production extrêmement élevés. Les technologies de culture cellulaire sont complexes, posent d'importantes difficultés lors de la transposition industrielle, et les délais de construction et de mise en service des unités de production sont longs.

\section{Eufs embryonnés}

Depuis 1937, le vaccin antigrippal est produit suivant un procédé de fabrication majoritairement inchangé, par l'inoculation d'œufs de

\begin{tabular}{|c|c|c|c|c|c|c|}
\hline Points à considérer & $\begin{array}{l}\text { Systèmes de } \\
\text { production }\end{array}$ & & & & & \\
\hline & Bactéries & Levures & $\begin{array}{c}\text { Cellule } \\
\text { d'insectes } \\
+ \\
\text { baculovirus }\end{array}$ & $\begin{array}{c}\text { Cellules } \\
\text { animales } \\
\text { (cellules } \\
\text { СНO) }\end{array}$ & $\begin{array}{c}\text { Plantes } \\
\text { transgéniques }\end{array}$ & $\begin{array}{c}\text { Animaux } \\
\text { transgéniques }\end{array}$ \\
\hline $\begin{array}{l}\text { Niveau de production } \\
\text { théorique }\end{array}$ & ++++ & ++++ & +++ & + & +++++ & +++++ \\
\hline Niveau de production pratique & $++(+)$ & $++(+)$ & + & + & ++ & +++ \\
\hline Coût d'investissement & ++++ & ++++ & ++ & + & +++ & +++ \\
\hline Coût de production & ++++ & ++++ & ++ & ++ & ++++ & +++ \\
\hline Flexibilité & ++++ & ++++ & ++ & + & ++++ & +++ \\
\hline Conservation & ++++ & ++++ & ++ & +++ & ++++ & ++++ \\
\hline Stabilité & +++++ & ++++ & +++ & +++ & +++++ & +++++ \\
\hline Délai pour la $1^{\text {re }}$ production & ++++ & +++++ & +++ & ++++ & ++++ & $+++(+)$ \\
\hline Augmentation & ++++ & ++++ & ++ & + & ++++ & ++++ \\
\hline Recueil & ++++ & ++++ & ++++ & ++++ & ++++ & +++ \\
\hline Effets sur l'organisme & $+++(+)$ & $+++(+)$ & $+++(+)$ & $+++(+)$ & $+++(+)$ & +++ \\
\hline $\begin{array}{l}\text { Modifications } \\
\text { post-translationnelles }\end{array}$ & + & ++ & +++ & +++ & +++ & ++++ \\
\hline Glycosylation & + & ++ & +++ & +++ & ++ & ++++ \\
\hline Stabilité du produit & ++++ & ++++ & +++ & ++ & +++ & +++ \\
\hline Purification & +++ & +++ & +++ & +++ & +++ & +++ \\
\hline Pathogènes contaminants & ++++ & ++++ & ++++ & ++++ & ++++ & +++ \\
\hline Propriété intellectuelle & ++++ & +++ & +++ & ++ & +++ & +++ \\
\hline Produits sur le marché & ++++ & +++ & +++ & +++++ & + & +++ \\
\hline
\end{tabular}

Tableau II. Comparaison entre les différents systèmes de production des protéines pharmaceutiques recombinantes. Source: L.M. Houdebine [10]. 


\section{Le Centre de Bioproduction à Genopole ${ }^{\circledR}$ : le premier centre de production GMP à façon de lots cliniques de protéines à visée thérapeutique en France}

\section{Interview d'Alain Métayer, Responsable du programme Bioproduction à Genopole ${ }^{\circledR}$}

La création d'un Centre de Bioproduction à Genopole ${ }^{\circledR}$ constitue une première étape décisive dans le développement industriel de Genopole ${ }^{\circledR}$ dans ce domaine et positionne celui-ci en référence française et européenne de la bioproduction.

Opérationnel en 2009, il produira des protéines recombinantes, notamment des anticorps monoclonaux sous statut GMP ${ }^{1}$ destinés aux essais cliniques pour les phases I et II. L'objectif est de satisfaire les besoins de production des entreprises de biotechnologies, des instituts et des laboratoires de recherche et d'offrir une alternative de production aux entreprises pharmaceutiques.

Dans le contexte actuel d'un marché biopharmaceutique en pleine expansion, les conclusions des études sur la bioproduction sont claires: les capacités de production de molécules biologiques, à usage thérapeutique notamment, sont insuffisantes pour satisfaire les besoins des entreprises de biotechnologie, des laboratoires de recherche et de l'industrie pharmaceutique. En 2007, la France ne disposait que de 3 centres de production à façon de petite taille, produisant de faibles quantités, dans des délais très longs et utilisant des techniques ne permettant pas l'accompagnement industriel global et cohérent jusqu'à I'AMM. Suite à la publication de l'étude « bioproduction » du LEદM en 2004, Genopole ${ }^{\circledR}$ prend la décision de créer un Centre de Bioproduction de protéines thérapeutiques en condition GMP pour le développement et la fabrication de protéines recombinantes par culture de cellules de mammifères. Sa mise en exploitation est prévue en 2009.

\section{Des enjeux forts}

Le Centre de Bioproduction doit contribuer à combler les besoins des sociétés de biotechnologies françaises et européennes, en produisant à façon des protéines recombinantes et des anticorps monoclonaux (molécules essentielles pour le traitement du cancer) sous statut GMP* destinés à réaliser des essais cliniques de phases I et II.

«La production à façon est la modalité la plus complexe à mettre en œuvre puisqu'elle suppose de pouvoir traiter successivement et parallèlement des biomolécules différentes en éliminant tout risque de contamination croisée. Elle nécessite donc de répondre à des exigences particulièrement draconiennes que constituent les GMP. Ce sera le premier centre de production à façon de cette taille en France, un des 10 premiers d'Europe. Face aux difficultés que rencontrent actuellement les entreprises de biotechnologies pour produire leurs molécules d'intérêt, il représente un outil industriel indispensable », explique Alain Métayer.

\section{Des objectifs ambitieux}

À court terme, l'objectif de Genopole ${ }^{\circledR}$ est de développer l'ensemble de la chaîne de valeur dans le domaine de la santé, en proposant une offre globale allant de l'ingénierie cellulaire à la répartition aseptique de produit. Afin d'offrir une alternative aux cellules animales, il est envisagé la création d'un deuxième centre de production de protéines à visée thérapeutique utilisant les levures et les bactéries, technologies éprouvées destinées à pro- duire des molécules plus simples, dans des délais plus courts. En amont, un centre-laboratoire d'ingénierie cellulaire et moléculaire prendra en charge le développement et l'optimisation du meilleur système d'expression pour une molécule donnée. Enfin, une unité de formulation et de répartition aseptique des lots cliniques complètera l'offre en aval.

"L'objectif est de proposer très vite aux clients une offre complète de techniques et services entièrement implantés à Évry, d'amont en aval de la production, de manière à donner à Genopole ${ }^{\circledR}$ une visibilité européenne en bioproduction, depuis la sélection du gène d'intérêt jusqu'à l'essai clinique. Certains essais pourront pour certaines pathologies être réalisés sur le site d'Évry, dans la cadre du Centre Hospitalier Sud-Francilien d'Évry-Corbeil, qui ouvrira en 2011 et auquel sera rattaché un centre de recherche clinique et translationnelle. On sait en effet que si les molécules partent à l'étranger dès la première phase clinique, elles y restent pour les phases suivantes et les lots commerciaux... », souligne encore Alain Métayer.

À plus court terme encore, un autre objectif sera d'opérer une diversification vers la production de protéines non thérapeutiques, pour la cosmétologie et la chimie fine, activité à façon qui, pour l'instant, n'existe pas significativement en France, pour la production de lots pré-industriels d'enzymes innovantes et de produits issus de procédés biocatalytiques originaux. Le Centre Enzymes et Biocatalyse créé à Genopole ${ }^{\circledR}$ devrait être opérationnel en 2010.

\section{Des perspectives en Recherche \& Développement}

Le développement de la bioproduction doit permettre de relever un défi industriel majeur : diminuer le prix de revient des biomédicaments grâce à des process de production innovants capables d'augmenter les rendements et d'abaisser les coûts. Cette problématique suscite d'importantes questions R\&D : comment augmenter la productivité des clones? Comment réduire le taux de perte de produits dans les process de purification? Comment réduire le temps d'occupation de chaque zone ? Comment développer l'usage unique pour supprimer les temps de nettoyage des équipements et augmenter la sécurité ? L'objectif final est de pouvoir produire plus vite et/ou davantage. Les unités de production qui y parviendront détiendront un avantage concurrentiel fondamental. Tous ces aspects, ainsi que leurs incidences notamment en termes de formation, étaient au programme du $2^{e}$ Symposium international de bioproduction organisé par Genopole ${ }^{\circledR}$ le 6 octobre 2008. 
Chiffres clés du Centre de Bioproduction

- Taille : $1300 \mathrm{~m}^{2}$, dont $700 \mathrm{~m}^{2}$ de salles blanches de production classées de $D$ à $B$.

- Capacité : 8 à 10 lots par an produits grâce à plusieurs salles de culture cellulaire et à une suite de bioréacteurs allant de 20 à 1250 litres, produisant de $100 \mathrm{~g}$ à $1 \mathrm{~kg}$ de protéine purifiée par lot.
- Process : 3 zones indépendantes de culture cellulaire pour la production simultanée de 3 biomolécules; une zone de purification en 3 étapes: 2 salles de chromatographie et une salle de nanofiltration. Toutes les salles de production possèdent un système de traitement de l'air indépendant, une chambre froide dédiée et un traitement individuel des rejets.

- Effectif : 15 à 20 personnes. poule embryonnés avec le virus Influenza. Ce procédé de production de vaccins sur œufs embryonnés est très complexe et se prête difficilement à une industrialisation massive. L'approvisionnement massif en œufs embryonnés reste un défi majeur, dans la mesure où ceux-ci doivent remplir des critères préétablis tels que l'épaisseur de la coquille, la taille, la charge microbienne, le nombre et la viabilité des embryons...

Cette méthode qui existe depuis plusieurs décennies est bien établie, les procédés de production sont validés et les unités de production accréditées par les autorités pharmaceutiques de tutelle. Le seul paramètre variable d'année en année est la souche du virus sélectionnée servant à l'inoculation des œufs.

Le Tableau /I résume les avantages et inconvénients des systèmes d'expression de protéines thérapeutiques éprouvés.

\section{...et des technologies innovantes encore à l'étude}

De nouveaux systèmes d'expression, tels que les cellules aviaires, les cellules d'insectes, les animaux et plantes transgéniques, l'expression transitoire chez les végétaux, les microalgues, sont les alternatives actuellement à l'épreuve.

\section{Cellules d'insectes}

L'expression de protéines recombinantes dans les cellules d'insectes (notamment lignée Sf9 de Spodoptera frugiperda) est possible via l'utilisation de vecteurs d'expression développés à partir de Baculovirus pour la modification des cellules. Ce système permet une bonne expression de la protéine. Les cellules d'insectes sont capables d'effectuer des opérations post-traductionnelles, notamment la glycosylation, proches de celles effectuées par les cellules de mammifères et de sécréter la protéine recombinante dans le milieu de culture. Toutefois, ce modèle, qui permet la production de petites quantités de protéines, reste sensible, notamment parce qu'il présente l'inconvénient de disposer d'un système protéolytique qui limite sa productivité en protéine cible.

\section{Cellules aviaires}

Un modèle d'expression en cellules aviaires (lignées $\varepsilon B x^{\circledR}$ ) a été développé par la société française Vivalis pour la production de vaccins et de protéines humaines recombinantes. Les lignées $\varepsilon B x^{\circledR}$, diploïdes et non tumorigènes qui ont été établies à partir de cellules souches embryonnaires de canard, présenteraient des capacités de prolifération en bioréacteur à de hautes densités cellulaires, en milieux de culture exempts de sérum bovin.

Ces lignées ont démontré leur susceptibilité à différents virus humains et animaux, ce qui leur ouvre des perspectives pour la production de vaccins. Elles expriment efficacement et sécrètent des protéines humaines recombinantes qui présentent un profil de glycosylation très proche du profil humain, notamment à faible teneur en fucose, ce qui est favorable à l'efficacité thérapeutique des anticorps monoclonaux. Les cellules aviaires font actuellement l'objet de cultures en bioréacteurs de quelques dizaines de litres. Même si elles s'avèrent plus productives que les cultures de cellules de mammifères, leur production industrielle subira les mêmes contraintes en termes de transposabilité, d'investissements et de délais de mise en service des outils de production.

\section{Plantes transgéniques}

L'utilisation de plantes transgéniques (tabac, luzerne, colza, maïs, riz, soja, pomme de terre...) pour la production de protéines thérapeutiques paraît au premier abord très intéressante, dans la mesure où la transgenèse est très facile. Les autres avantages de la production dans les plantes transgéniques sont le faible prix de revient de la production, une grande flexibilité pour suivre les demandes du marché, de faibles investissements de départ et une totale sécurité par rapport aux risques infectieux (virus, prions). Cependant, les protéines produites, stockées préférentiellement dans les feuilles ou les graines, peuvent être difficiles à extraire et à purifier, et les extraits peuvent contenir des substances (protéases, polyphénols...) mal tolérées par I'homme. Les cellules végétales étant des eucaryotes, elles disposent d'une machinerie cellulaire complexe et sophistiquée qui leur permet de réaliser des modifications post-traductionnelles très proches de celles des mammifères. Toutefois, les protéines produites sont dépourvues de terminaisons acide sialique et, comme chez les insectes, la glycosylation reste incomplète. De plus, elle ajoute aux protéines des résidus xylose qui pourraient être immunogènes.

C'est avec les problèmes éthiques et environnementaux posés par la culture des plantes transgéniques, le principal écueil de ce système. De nombreux travaux sont en cours pour modifier les capacités de glycolysation des protéines par les plantes, et demanderont encore 
plusieurs années de développement, en vue de produire des protéines thérapeutiques administrables par voie parentérale. Cependant, dans la mesure où, de par son alimentation, l'homme est constamment exposé aux glycoprotéines végétales, l'administration de protéines recombinantes produites par le système végétal pourrait être envisagée par les voies orales et topiques, nécessitant toutefois des galéniques complexes pour préserver leur activité dans le tractus digestif et, le cas échéant, leur permettre de passer la barrière intestinale. L'administration par voie orale est tout particulièrement adaptée dans le cas des pathologies du tractus digestif. Citons les études cliniques qui ont été menées par la société française Meristem ${ }^{\circledR}$ Therapeutics sur la lipase gastrique recombinante, produite dans les grains de maïs transgénique, administrée par voie orale pour le traitement de troubles nutritionnels sévères (malabsorption des graisses) liés à la mucoviscidose. L'utilisation de plantes alimentaires transgéniques est également envisagée pour le développement de vaccins oraux, administrés directement sous forme d'aliments ou nécessitant une galénique adaptée. On citera notamment l'ingestion par les souris de pomme de terre exprimant un antigène de surface du virus de I'hépatite $B$ qui déclenche une réponse immunitaire. La production d'une sous-unité de la toxine du choléra a été également réalisée dans la pomme de terre, et une glycoprotéine du virus de la rage à été produite dans une tomate transgénique, premier exemple d'un vaccin comestible.

Plusieurs pistes sont proposées pour remédier aux problèmes environnementaux liés à l'utilisation de plantes transgéniques - dissémination non contrôlée des transgènes par le pollen, voire des protéines cibles - quand les plantes sont cultivées en plein champ. Il est possible par exemple d'utiliser des plantes stériles pour éviter la dissémination du transgène ou des plantes qui ne font pas partie des aliments humains, comme la luzerne ou le tabac. Une autre possibilité est de cultiver les plantes en environnement confiné (serres répondant à des contraintes de confinement biologique spécifiques), bien que cela augmente notablement les coûts de production et diminue la flexibilité et l'extensivité de la production agricole.

\section{Expression transitoire dans des plantes non-0GM}

La production de protéines thérapeutiques par expression transitoire dans les plantes présente les avantages du modèle végétal, sans les inconvénients des cultures d'OGM. Le cycle de production de biomasse végétale, de quelques semaines, est réalisé avec des plantes non modifiées. À leur stade optimal de croissance, elles seront soumises à la technologie d'agro-infiltration, qui consiste en leur contamination, par le vecteur d'expression modifié Agrobacterium tumefaciens (bactérie phytopathogène, responsable de la galle du collet chez les végétaux), porteur du ou des gènes d'intérêt codant pour la production de protéines recombinantes. Au bout d'une semaine, les plantes, qui ont ainsi synthétisé et accumulé la molécule cible, vont rentrer dans le procédé d'extraction/purification. L'expression est dite transitoire, dans la mesure où l'exogène n'est pas intégré au génome de l'organisme hôte et où elle est perdue en général à la prochaine mitose.

Les productions végétales doivent être menées hors sol, afin de pouvoir soumettre les plantes à la technologie d'agro-infiltration, ce qui ne permet pas, en l'état actuel de la technologie, d'envisager des productions de type grandes cultures. Cependant, d'excellents résultats en termes de productivité permettent de produire des quantités importantes de protéines recombinantes sur des surfaces de quelques milliers de $\mathrm{m}^{2}$, rapidement et à faible coût, avec un investissement industriel limité.

Ce modèle est actuellement développé par la société canadienne Medicago pour la production de vaccins pandémique et saisonnier contre la grippe. L'hémagglutinine, protéine de surface du virus Influenza, est synthétisée par Nicotiana benthamiana (famille du tabac) sous la forme de particules pseudo-virales, structures sphériques produites par bourgeonnement de la membrane plasmique végétale et présentant les molécules d'hémagglutinine en leur surface, à la façon du virus.

Dans le domaine des vaccins, le potentiel immunogène des protéines recombinantes présentant une glycosylation végétale pourrait représenter un avantage. De même, les lipides membranaires des particules pseudo-virales d'origine végétale sembleraient favoriser la réponse immunitaire cellulaire chez les sujets vaccinés.

\section{Micro-algues}

Les micro-algues, réservoir inexploité de molécules thérapeutiques, suscitent depuis des décennies l'intérêt et la curiosité des chercheurs. Depuis une quinzaine d'années, leur utilisation comme système d'expression de protéines recombinantes est explorée. Qu'il s'agisse d'espèces procaryotes (cyanobactéries) ou eucaryotes, photo-autotrophes ou hétérotrophes, le challenge pour leur production en masse est technologique. En effet, la production de protéines thérapeutiques nécessite que les micro-algues puissent se développer en culture monospécifique et confinée, c'est-à-dire en système clos. La mise au point de photobioréacteurs tubulaires requiert des investissements industriels importants pour des espèces à faible taux de croissance (temps de doublement minimum de 2 jours, contre 20 minutes pour certaines bactéries). Les progrès technologiques en termes de conception de bioréacteurs de type poches à usage unique pourraient cependant ouvrir de nouvelles perspectives, bien que les volumes utiles en soient limités à quelques centaines de litres. La production de protéines recombinantes par des micro-algues hétérotrophes en fermenteur, quant à elle, ne pourrait concurrencer les technologies hautement maîtrisées à partir de microorganismes industriels que sont les bactéries et levures. 
L'extraction et la purification de protéines de la biomasse microalgale constituent, elles aussi, un défi. En effet, les espèces de micro-algues fortement productrices de protéines ne les sécrètent pas dans le milieu; en revanche, elles sécrètent des polyssacharides en très grande quantité, ce qui de toute façon rendrait complexe la purification d'éventuelles molécules sécrétées, du fait, entre autres, de problèmes de viscosité. L'extraction de protéines intra-cellulaires, quant à elle, reste également complexe, les cellules micro-algales étant le plus souvent difficiles à éclater et les contenus cellulaires très chargés. Certaines Rhodophycées (algues rouges) sont par exemple capables d'accumuler des pigments protéiques (phycobiliprotéines) représentant jusqu'à $15 \%$ de la matière sèche, ce qui rend complexes les opérations d'extraction/purification.

Du fait de ces obstacles technologiques, les micro-algues continuent d'apparaître plus comme des réservoirs génétiques et moléculaires pour la drug discovery que comme des modèles d'expression de molécules recombinantes.

\section{Les animaux transgéniques}

L'utilisation d'animaux transgéniques présente l'avantage de fournir des protéines de grande qualité dans le lait, le sang ou le plasma séminal. La difficulté réside dans la séparation des protéines de l'animal de celles que l'on fait produire et une attention spéciale doit être portée à la possible présence de pathogènes au sein des protéines produites.

Le système le plus avancé est la production de protéines dans le lait des animaux transgéniques. Preuve en est l'autorisation de mise sur le marché par l'EMEA (European Agency for the Evaluation of Medicinal Products) d'un premier produit en 2006, I'ATryn ${ }^{\circledR}$ - antithrombine III humaine produite dans le lait de chèvres transgéniques par la société américaine GTC Biotherapeutics. D'autres produits sont en cours de développement, des protéines plasmatiques notamment, qui pourraient rencontrer le même succès dans les 5 à 10 prochaines années.

Les animaux transgéniques pourraient devenir une alternative pertinente pour produire des protéines difficiles à exprimer en cellule, ou pour fabriquer à moindre coût des biosimilaires de produits existants. Quant au choix de l'espèce, c'est le facteur temps qui est le critère principal. La durée des cycles reproductifs des animaux de grande taille n'est pas compatible avec le développement pharmaceutique. Si la vache apparaît comme l'animal le plus intéressant en termes de volumes laitiers et de maîtrise des technologies de fractionnement du lait, ses durées de maturation sexuelle puis de gestation sont peu compatibles avec le cycle de développement d'un médicament. Ainsi, il faut au minimum 36 mois pour obtenir les premiers laits à partir d'une vache transgénique, contre à peine un an pour une lapine.

Le modèle chèvre semble présenter le meilleur rapport entre la durée de gestation, les volumes de lait produits et le degré de mécanisation des technologies d'élevage et de traite. Pour les petites quantités de produits (jusqu'à $10 \mathrm{~kg}$ ), le modèle lapine demeure pertinent.

Un cheptel de lapines transgéniques pourrait ainsi être suffisant pour alimenter les marchés de niche. Le lapin, modèle animal de laboratoire, présente l'avantage majeur de pouvoir être élevé avec un statut sanitaire contrôlé de type EOPS (exempt d'organisme pathogène spécifique). Ce statut n'existe pas pour les animaux plus gros. Le lapin est également réfractaire aux maladies à prions, ce qui n'est pas le cas des ruminants.

Bien que l'utilisation des mammifères reste le système le plus éprouvé, notamment parce que les problèmes de glycosylation sont mineurs, l'utilisation d'animaux non-mammifères, comme les espèces aviaires (production dans le blanc d'œuf) devenues attractives grâce à l'amélioration des méthodes d'obtention des oiseaux transgéniques, est une autre voie. Deux anticorps monoclonaux et l'interféron $\beta$ la humain ont été obtenus grâce à ce modèle, qui pose cependant deux difficultés principales: la non-transmission du transgène à la descendance, d'une part, et des profils de glycosylation différents de l'homme qui entraînent des problèmes d'immunogénicité et d'efficacité, d'autre part.

\section{Vers un système idéal ?}

Il apparaît très clairement que la production de protéines à partir de bactéries et de levures demeure le système par excellence pour l'obtention de molécules simples car il reste très compétitif.

Les systèmes utilisant des cellules de mammifères, bien que très coûteux, demeurent très étudiés, et de gros efforts sont portés à l'amélioration de la productivité des lignées, dans la mesure où ils permettent de produire des molécules complexes à potentiel thérapeutique élevé.

Les technologies innovantes se situent à une étape charnière où l'on devrait assister au début d'un développement industriel à grande échelle. Le succès de ce développement dans les cinq prochaines années sera déterminant pour l'avenir commercial de ces technologies. Au stade de développement actuel, ce succès repose moins sur la réussite technique que sur la capacité qu'auront les entreprises qui développent ces technologies à créer de l'intérêt et à obtenir des ressources financières adéquates. Dans un premier temps, ces technologies devraient évoluer et éventuellement remplacer les technologies actuelles, en s'établissant dans certaines niches où elles possèdent des avantages uniques et difficilement égalables par les technologies courantes (rapidité, coûts de production, protéines difficiles à produire dans d'autres systèmes).

Le modèle «cellules aviaires» semble pouvoir représenter une alternative technologique intéressante et assez mature, surtout pour la production de vaccins mais aussi pour celle d'anticorps monoclonaux. 
Le passage au niveau industriel des systèmes utilisant des plantes ou animaux transgéniques nécessite une évolution des mentalités, compte tenu de la méfiance que génèrent les organismes génétiquement modifiés dans certains pays, notamment en France.

Les modifications post-traductionnelles, comme la glycolysation, sont importantes pour la synthèse des protéines thérapeutiques qui sont pour la plupart des glycoprotéines. Les sucres représentent une grande proportion des glycoprotéines et sont déterminants dans leur structure et donc dans leur activité. C'est pourquoi, ces dernières années ont vu l'avènement de nouvelles technologies de modification de la glycosylation des protéines recombinantes, entraînant une meilleure compréhension de la relation glycosylation/ effet thérapeutique.

Il apparaît aujourd'hui que la glycosylation humaine n'est pas forcément indispensable pour un effet thérapeutique donné et que la question de la nécessité d'une glycosylation la plus proche possible de celle réalisée chez l'Homme doit se poser au cas par cas, en fonction de l'indication thérapeutique ciblée.

Dans ce contexte, les systèmes tels que les plantes, dans lesquelles la glycosylation devrait pouvoir être contrôlée facilement et de façon efficace, ont un avantage, même si ce système reste actuellement assez éloigné du marché. Dans le domaine plus spécifique des vaccins, leur potentiel immunogène pourrait également représenter un avantage. Dans tous les cas, la capacité de modifier la glycosylation dans les plantes - y compris la possibilité de supprimer les glycans spécifiques des plantes - sera certainement bénéfique pour le développement futur de cette technologie.

Le système idéal, s'il devait, ou pouvait exister, serait certainement un modèle permettant d'intégrer une ou plusieurs copies du gène d'intérêt dans un seul locus bien défini et autorisant un haut niveau de production. Le transgène serait stable génétiquement, sans pression de sélection dans le milieu de culture. Ce modèle idéal ne serait susceptible de transmettre aucun pathogène (virus, prion) à l'homme. En effet, l'environnement réglementaire de la sécurité virale au cours du procédé de purification a un impact très significatif sur le coût de production. Enfin, ce modèle serait de mise en œuvre rapide, et nécessiterait des investissements industriels limités, que ce soit pour la production, la purification ou la mise sous forme pharmaceutique. $\diamond$

\section{SUMMARY}

Bioproduction of pharmaceutical proteins:

review and perspectives

The increase in importance of the biopharmaceutical market generates enormous needs in production of therapeutic molecules. The world capacities of production, mainly therapeutic proteins, impossible to obtain by chemical synthesis, are at present insufficient. Besides the economic aspects in set, play, the question of the technologies used to produce these molecules is important: the tried(felt) technologies (bacteria, yeasts, cells of mammals), used for a long time rather widely remain expensive, or see their limited field of application; the innovative technologies (avian cells, animal and plant transgenic, among others), as for them, again have to show their ability. $\diamond$

\section{REMERCIEMENTS}

Les auteurs remercient Alexandre Fouassier (Bioprotein Technologies), Brigitte Barbeau et Frédéric Ors (Medicago), et Olivier Cochet (Pierre Fabre), pour avoir, en répondant à quelques questions, considérablement enrichi le propos de cet article.

\section{BIBLIOGRAPHIE}

1. Étude «Bioproduction 2008, état des lieux et recommandations pour l'attractivité française » conduite par le LદદM. Paris : Les Entreprises du Médicament et Genopole, 2008.

2. Blanchard S. Ingénierie de glycoside hydrolases pour la glycosylation des protéines recombinantes. Grenoble 1: Thèse de doctorat-Université Joseph Fournier, $2004: 230 \mathrm{p}$.

3. Desgranges C. Anticorps monoclonaux et thérapeutique. Pathol Biol $2004 ; 52$ : 351-64.

4. Faye L, Landry N, Lerouge P, Gomord V, Vézina LP. La production de protéines à usage biopharmaceutique dans les plantes. Med Sci (Paris) $2001 ; 17: 867-77$.

5. Gomord V, Chamberlain P, Jefferis R, Faye L. Biopharmaceutical production in plants : problems, solutions and opportunities. Trends Biotechnol $2005 ; 23: 559-65$.

6. Houdebine LM. Production of pharmaceutical proteins by transgenic animals. CIMID 2008 ; $647: 1-15$

7. Kermode AR. Plants as factories for production of biopharmaceutical and bioindustrial proteins: lessons from cell biology. Canad J Botany 2006 ; $84: 679-94$.

8. Ma S, Jevnikar AM. Transgenic rice for allergy immunotherapy. Proc Natl Acad Sci USA 2005; $102: 17255-6$.

9. Saint-Jore-Dupas C, Faye L, Gomord V. From planta to pharma with glycolsylation in the toolbox. Trends Biotechnol $2007 ; 35: 317-23$.

10. Houdebine LM. Production of pharmaceutical proteins by transgenic animals. CIMID 2008 ; $647: 1-15$.

TIRÉS À PART

J. Dumas et B. Robert 